\title{
Experimental Verification of the Hybrid Magnetic Bearing Operation
}

\author{
Jan Vitner, Jirí Pavelka, Jiri Lettl \\ Department of Electric Drives and Traction \\ Faculty of Electrical Engineering, CTU in Prague \\ Prague, Czech Republic \\ jan.vitner@cvut.cz
}

\begin{abstract}
This paper discusses the proposal, realization, controlling system implementation and function verification of a hybrid magnetic bearing. The hybrid magnetic bearing in comparison with active and passive magnetic bearings exploits both passive and active magnetic fluxes. This type of bearing is not given too much attention in the literature despite of its many advantages. The issue of active and passive magnetic bearings is widely elaborated against the issue of hybrid magnetic bearings. The hybrid magnetic bearing is the special kind of a radial magnetic bearing. This magnetic bearing exploits permanent magnets to produce the basic magnetic flux acting the rotor and 3-phase double layer winding to influence this flux by an active magnetic flux. Basically, the hybrid magnetic bearing was developed for high speed bearing-less devices. According to that, we are expecting the achievement the highest rotor levitation stability in wide range of rotational speed and in various driving modes. The quality of the levitation is evaluated by international standards [ISO 14839]. In this paper, results of experimental verification of the levitation stability are presented.
\end{abstract}

Keywords - magnetic bearing; positional stabilization; magnetic levitation; power electronic

\section{INTRODUCTION}

Magnetic bearings have been found very applicable for suspending high-speed rotary systems, such as flywheel systems [1], vacuum pumps or turbocompressors [2]. They are being increasingly used in applications where minimum friction is desired or in chemically aggressive environments where traditional bearings are unacceptable for their lubrication system or other features [3]. Passive magnetic bearings (PMB) feature very little loss due to no current, but have no active control ability and low damping stiffness [4]. Active magnetic bearings (AMB) have the control ability and high stiffness characteristic, but the existence of biased current brings power losses [5-6]. Therefore, more attention is paid to the permanent magnet biased hybrid magnetic bearing (HMB), which combines the merits of $\mathrm{PMB}$ and $\mathrm{AMB}$.

Hybrid magnetic bearings, while usually being more complex to construct, require smaller ampere-turns per unit force than equivalent active magnetic bearings, have higher sensitivity as a function of the drive coil current, usually exhibit better linearity as a function of the drive coil current, and can be made more compact. If the rotor is laminated, they can also provide lower rotor hysteresis and eddy current losses. Since HMB bearings can operate in the virtual zero power mode (VZP), where the static bearing load is supported by the permanent magnet field, thus they can operate with very low energy consumption.

Most of the previously published works deals with levitation control adjustment of the active magnetic bearing [7-8], whereas this paper is primarily focused on the control system adjustment of the real permanent magnet based active magnetic bearing with the 3-phase stator winding [9].

The chapter II. of this paper describes the structure of the HMB and fundamental function of its parts. Following chapter III. deals with mathematical description of the two major forces acting the rotor and their significant features. The simulation (see chapter IV.) of the HMB behavior is followed by chapter $\mathrm{V}$. with description of the realized system and with the presentation of the HMB levitation measurement results.

\section{DESCRIPTION OF THE HYBRID MAGNETIC BEARING}

The magnetic circuit is assembled from two identical parts with above mentioned winding in each part. Eighteen permanent magnets are placed around the perimeter between both parts [10], as shown in Fig. 1.

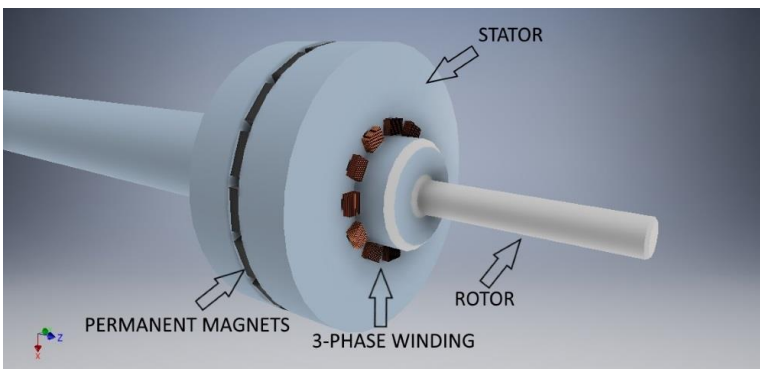

Figure 1. View of the whole hybrid magnetic bearing

The 3-phase winding creates a source for magnetic circuit (red line in Fig. 2) and produces controlled magnetic field in this circuit. This active magnetic field is closing in radial plane. Magnetic resistivity of the magnetic circuit for this active magnetic field is independent on the rotor shaft motion. The active magnetic field flows through both horizontally 
opposite air gaps and therefore the sum of both air gap lengths is constant.

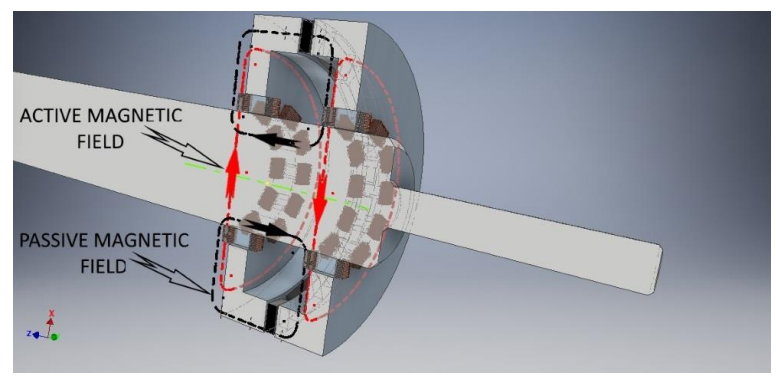

Figure 2. Cut of the magnetic circuit of the hybrid magnetic bearing

The second magnetic source is passive without any possibility to control its magnetic force. All permanent magnets are polarized along the whole stator circumference in the same direction. The path of the passive magnetic field leads from the north pole of permanent magnet in the axial plane to the stator teeth, then through the air gap to the rotor and continues along the axis of the rotor to the vertically opposite stator teeth through the air gap to the south pole of the permanent magnet (black line in Fig. 2). Magnetic resistivity of the magnetic circuit of the passive magnetic field depends on the rotor position in the air gap. According to that, the permanent magnets are continuously attracting the rotor around the whole circumference and the attracting force is the higher the closer the rotor is to the stator.

The view of the real HMB placed in the laboratory of CTU in Prague shows the Fig. 3.

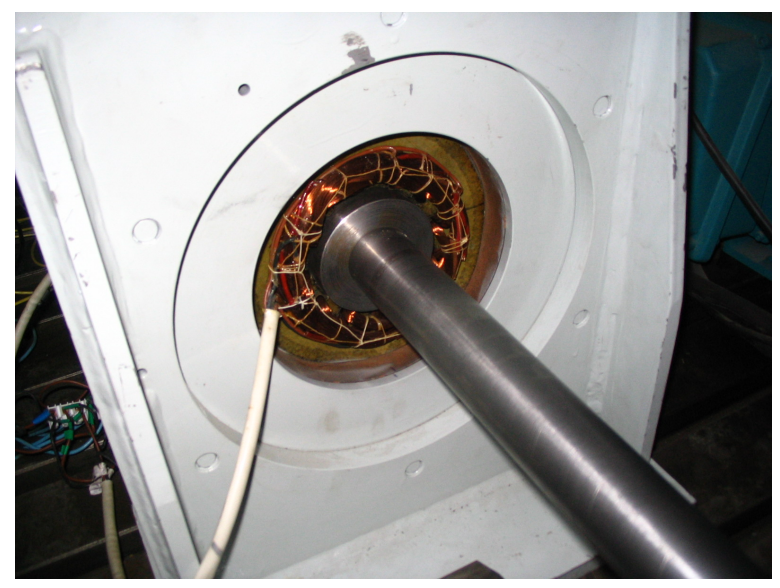

Figure 3. Hybrid Magnetic Bearing

\section{PASsive AND ACTIVE MAGNETIC FIELDS}

In order to create the right mathematical model, it is necessary to verify, numerically determine and to gauge parameters of the stator magnetic circuit and the used permanent magnets. Via such mathematical model it is possible to calculate the force effects of the passive magnetic field on the rotor for any deviation. The magnetic resistance of each part of the magnetic circuit is given by:

$$
R_{m i}=\frac{1}{\mu_{0}} \times \frac{l_{i}}{\mu_{i} \times S_{i}}[A t / W b]
$$

\section{A. Passive Magnetic Field}

According to the calculation of magnetic resistance for each part of the magnetic circuit, it is clear, that the magnetic resistance of iron is approximately $13 \backslash \%$ ampere-turns for the air gap. It will be considered in the coefficient of the next equation. The relation between the magnetic flux and the magneto-motoric force for the whole magnetic circuit at the various $R_{m \delta}$ is given by:

$$
F_{m}=\left(0.13 \times R_{m \delta 0}+R_{m \delta}\right) \times \Phi \quad[N]
$$

The magnetic induction under any stator pole is given by the total magnetic flux and the total area of all the stator teeth:

$$
B_{\delta 1 z}=\frac{\Phi}{S_{\delta}}[T]
$$

The force, excited by the magnetic induction $\mathrm{B}_{\delta}$ under one tooth, is given by:

$$
F_{\delta}=\frac{1}{2} \times \frac{B_{\delta}^{2}}{\mu_{0}} \times S_{\delta} \quad[N]
$$

According to the above-mentioned equations, there is an important characteristic which describes the dependence of the force of the passive magnetic field acting the rotor as follows the deviation magnitude (Fig. 4).

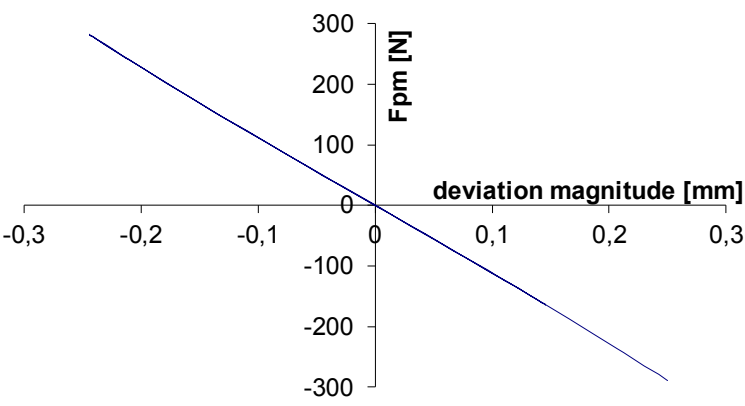

Figure 4. Dependence of the force of the permanent magnets on the air gap size

\section{B. Active Magnetic Field}

The magnetic resistance of the air gap for the active magnetic field is constant at any rotor position. According to the calculation of magnetic resistance at each part of magnetic circuit, it is clear, that the magnetic resistance of iron is approximately $22 \%$ ampere-turns for the air gap. It will be considered in the coefficient of the next equation. The relation between the magnetic flux and the magneto-motoric force for the whole magnetic circuit for the constant $\mathrm{R}_{\mathrm{m} \delta}$ is given by:

$$
F_{m w}=1.22 \times R_{m \delta} \times \Phi \quad[A t, m W b]
$$


According to the modified relation for the magneto-motoric force of the multi-phase winding we can calculate the corresponding current:

$$
F_{m w}=\frac{m \times N \times k_{v}}{\pi \times p} \times I \quad[N]
$$

The force of the exciting winding is dependent on the magnetic induction for 24 teeth on the stator:

$$
F_{w}=\frac{1}{2} \times \frac{B_{\delta}^{2}}{\mu_{0}} \times 2 \times 12 \times S_{\delta} \quad[N]
$$

Hence with the help of few transformations we can determine the required value of the required stator current in dependence on the required force (Fig. 5).

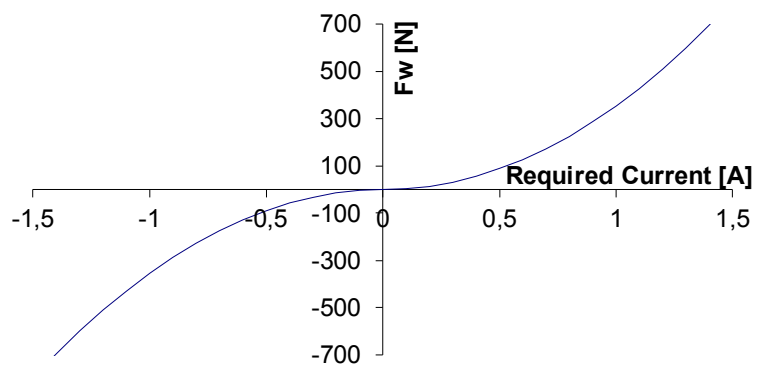

Figure 5. Dependence of the winding force on the required current

\section{SiMULATION OF THE HMB BEHAVIOR}

There is a simulation made in Matlab/Simulink. It was the first possibility to ensure, that such kind of concept is able to levitate. The basic block diagram is shown in Fig. 6.

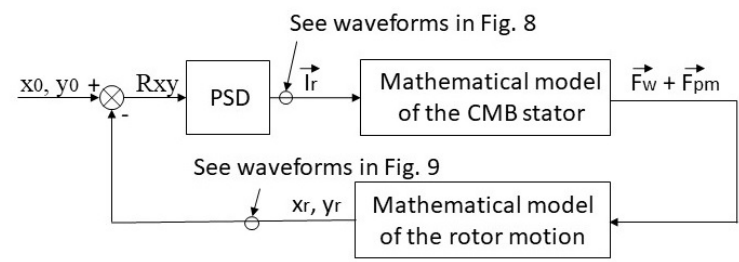

$\overrightarrow{\mathrm{Ir}}$ - required vector of stator current

$\vec{F}_{\mathrm{w}}, \overrightarrow{\mathrm{Fp}} \mathrm{p}$ - forces of winding and permanent magnets

$\mathrm{x}_{0}, \mathrm{y}_{0}-$ required rotor coordinates

$x_{r}, y_{r}-$ measured rotor coordinates

$\mathrm{R}_{\mathrm{xy}}$ - rotor deviation vector

Figure 6. The block diagram of the simulation routine

In Fig. 8 and Fig. 9 is shown the result of the simulation according to the right adjustment of all constants of the PSD and PS controllers [11-12]. Fig. 8 shows the PSD controller output in time and the Fig. 9 shows the position of the rotor. The real Matlab/Simnulink block diagram used to generate above mentioned outputs is depicted in Fig. 7.

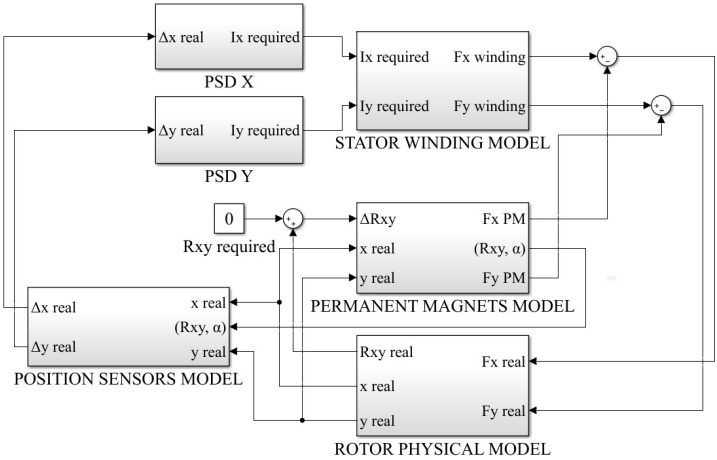

Figure 7. Matlab/Simulink model diagram

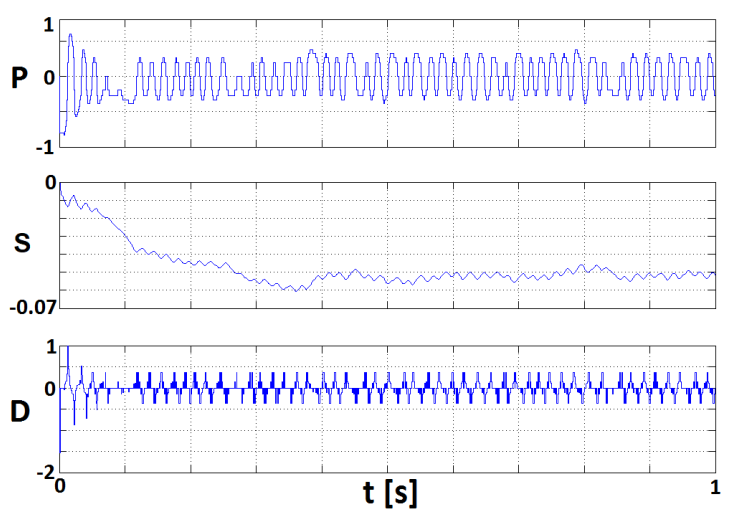

Figure 8. The course of the PSD components during the stabilization process

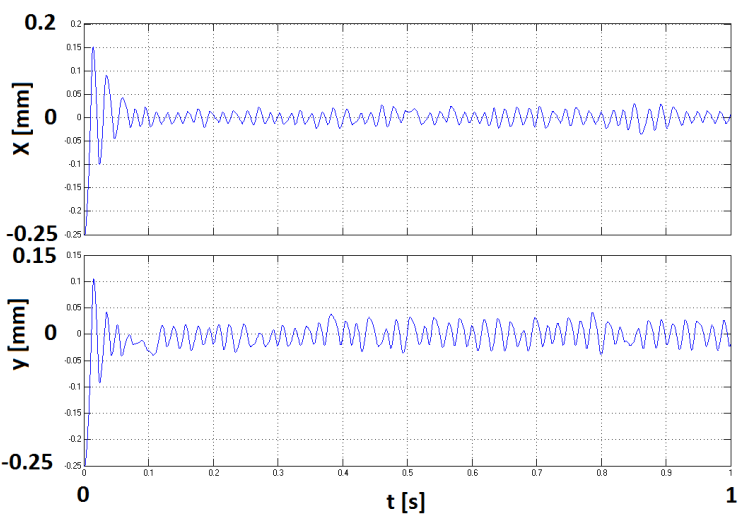

Figure 9. Dependence of the rotor position on time during the stabilization process

\section{RESUlTS OF EXPERIMENTAL MEASUREMENTS}

To stabilize position of the rotor in the center of the stator is the general point and the required target of our effort. The information about the actual position of the rotor is provided by two position sensors placed in mutual perpendicular axes $(\alpha, \beta)$. The information about the actual position of the rotor from position sensors is represented by two voltage signals that are converted by $\mathrm{A} / \mathrm{D}$ converters of the control unit. The digital information about the actual position of the rotor is an input for the PSD controller. The output of the PSD controller consists of two estimated values of stator current in axes $(\alpha, \beta)$. These two values of the stator estimated currents must be transformed to the 
real currents of the 3-phase stator system. Thus, we receive the estimated value of the current for each phase of the stator winding. The experimental setup of the system is illustrated in the Fig. 10.

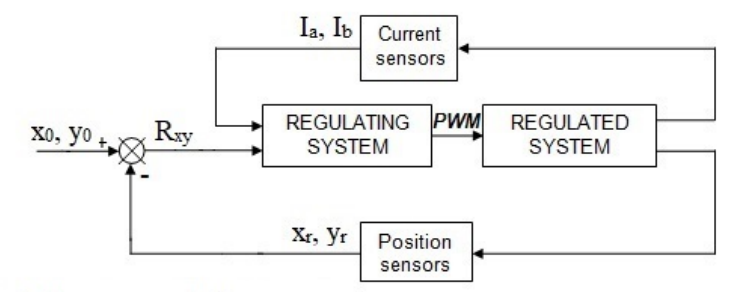

$\mathrm{I}_{\mathrm{a}}, \mathrm{I}_{\mathrm{b}}-$ measured phases currents

PWM - pulse width modulation duty cycle

$\mathrm{x}_{0}, \mathrm{y}_{0}$ - required rotor coordinates

$\mathrm{x}_{\mathrm{r}}, \mathrm{y}_{\mathrm{r}}-$ measured rotor coordinates

$\mathrm{R}_{\mathrm{xy}}$ - rotor deviation vector

Figure 10. Experimental setup block diagram

Stator windings are star connected and therefore the sum of all three instantaneous current values must be zero. Two from three stator phases have their own PS controller and the third current is given from the above-mentioned condition. Reliability and stiffness of the rotor stabilization is largely dependent on the achievable speed of the current PS controller.

The delta connection of stator phases and the parallel connection of windings in both sides of the stator winding were tested with the target to decrease the time constant of the current loop. The steady state value of the phase current was twice increased using the parallel connection. The achievable maximal current change in time is shown in Fig. 11 and its value is $4 \mathrm{~A} / \mathrm{ms}$.

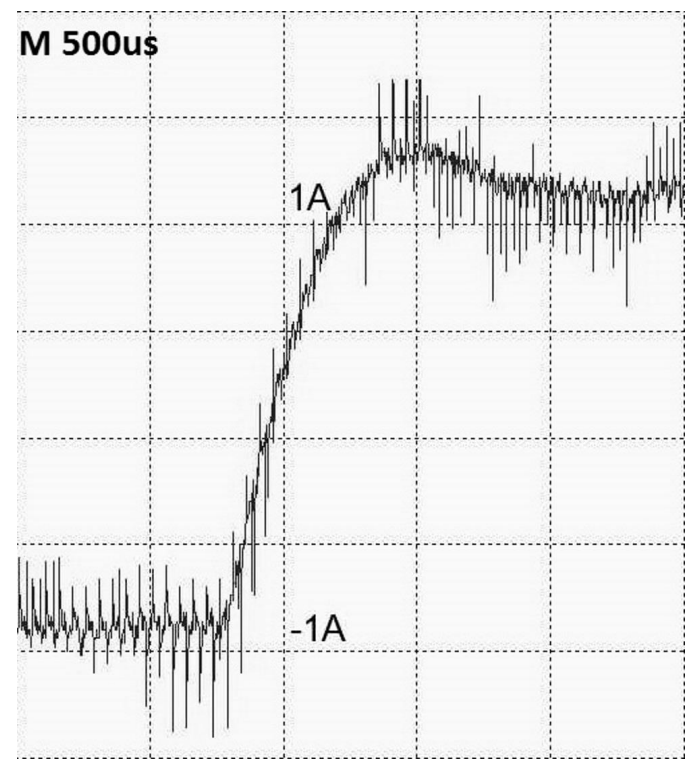

Figure 11. Phase current controlled by PS regulator

Before tuning the stabilization process, the rotor of the magnetic bearing had to be mechanically centered in the bore of the magnetic bearing stator as precisely as possible. In the next step, the rotor was mechanically fixed in the center of the horizontal axis and the stabilization process was tuned only for the vertical axis of the rotor motion. The system operates correctly only when we find the correct steady weight compensation value of the force acting the rotor. Without the steady weight compensation force, the special adjustment of the PSD controller would have to be performed for the vertical axis. Further, the rotor was fixed in the center position for the vertical axis and the rotor could move only in the horizontal axis. The stabilization process was successful with the similar adjustment of the PSD controller as was used for the vertical axis. In the last step, the rotor was released in both axes and stabilized in the center position of the stator bore. The levitation of the rotor is shown in Fig. 12.

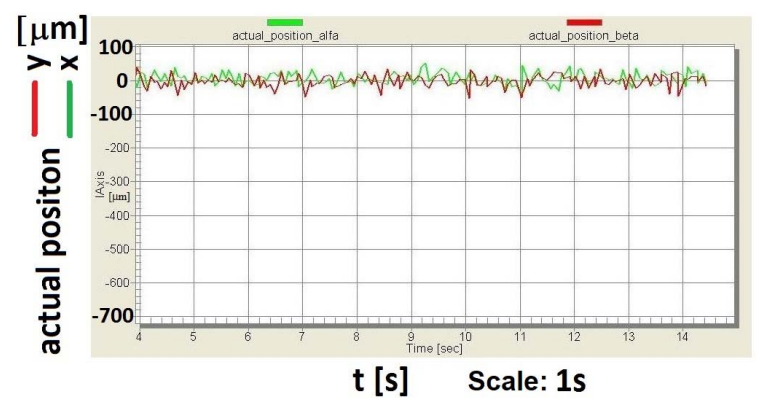

Figure 12. Levitation of the rotor around center of the stator bore

The air gap between the stator and rotor is $0.5 \mathrm{~mm}$ and during the stabilization process the magnitude of the rotor oscillations around the center position is approximately $\pm 50 \mu \mathrm{m}$.

\section{CONCLUSIONS}

Previous paragraphs summarize principle of the hybrid magnetic bearing. The view on the special kind of the magnetic bearing using both active and passive magnetic fields (permanent magnet based active magnetic bearing) is presented. Based on simulation and measurement on the designed and realized magnetic bearing system, the paper gives description of behavior, characteristics, and function of the system in steady states as well as in transient processes. Both simulation (chapter IV.) and experimental results (chapter V.) prove the functionality of the controlled system at linearization of the interaction between active and passive magnetic fields. The results in Fig. 9 and in Fig. 12 provide comparable outputs.

\section{ACKNOWLEDGMENT}

This paper was supported by the Technology Agency of the Czech Republic under the grant project No. TE02000103.

\section{REFERENCES}

[1] L. Shuliang, A. Palazzolo, Control of Flexible Rotor Systems with Active Magnetic Bearings. Journal of Sound and Vibration, July 2008, Vol. 314, Issues 1 and 2.

[2] R. Tiwari, A. Chougale, Identification of Bearing Dynamic Parameters and Unbalance States in a Flexible Rotor System Fully Levitated on Active Magnetic Bearings. Mechatronics, April 2014, pp. 274-286, Vol. 24.

[3] G. Schweitzer, E. H. Maslen, Magnetic Bearings. SpringerVerlag Berlin Heidelberg, 2009. 
[4] A. Chiba, T. Fukao, O. Ichikawa, M. Oshima, M. Takemoto, G. D. Dorrell, Magnetic Bearings and Bearingless Drives. 2005.

[5] Z. Sun, J. Zhao, Z. Shi, et al., Soft Sensing of Magnetic Bearing Systm Based on Support Vector Regression and Extended Kalan Filter. Mechatronics, April 2014, pp. 186197, Vol. 24.

[6] S. Cheng, M. W. Olles, A. F. Burger, et al., Optimization of a Hybrid Magnetic Bearing for a Magnetically Levitated Blood Pump via 3-D FEA. Mechatronics, October 2011, pp. 1163 1169, Vol. 21, Issue 7.

[7] S. Bouaziz, N. B. Messaoud, M. Mataar, et al., A Theoretical Model for Analyzing the Dynamic Behavior of a Misaligned Rotor with Active Magnetic Bearings. Mechatronics, September 2011, pp. 899-207, Vol. 21, Issue 6.
[8] Y. Liu, N. Tsai, H. Chiu, Construction of Rotor/AMB Dynamic Model Applied for TMP Using Self-tuning Kalman Filter. Mechatonics, March 2014, pp. 158-167, Vol. 24.

[9] ISO 14839-1, Mechanical vibration, Vibration of rotating machinery equipped with active magnetic bearings. Part 1: Vocabulary

[10] M. Kiani, H. Salarieh, A. Alasty, et al., Hybrid Control of a Three-Pole Active Magnetic Bearing. Mechatronics, November 2016, pp. 28-41, Vol. 39.

[11] M. H. Kimman, H. H. Langen, R. H. Munnig Schmidt, A Miniature Milling Spindle with Active Magnetic Bearings. Mechatronics, March 2010, p. 224-235, Vol. 20, Issue 2.

[12] Chi Zhang, K. J. Tseng, Design and Control of a Novel Flywheel Energy Storage System Assisted by Hybrid Mechanical-magnetic Bearings. Mechatronics, April 2013, pp. 297-309, Vol. 23, Issue 3. 